\section{Matriz Avaliativa do Vínculo Longitudinal na atenção primária em saúde: validação estatística em um território de saúde do Município do Rio de Janeiro, Brasil}

\author{
Evaluative Matrix of the Longitudinal Bond in \\ primary healthcare: statistical validation in a \\ health territory in the city of Rio de Janeiro, Brazil
}

\author{
Matriz Evaluativa del vínculo longitudinal en la \\ atención primaria en salud: validación estadística \\ en una zona con servicios de salud del Municipio \\ de Río de Janeiro, Brasil
}

Elenice Machado da Cunha 1 José Muniz da Costa Vargens 1 Marcio Candeias Marques 1 Gabriela Rieveres Borges de Andrade 2 Gisele O'Dwyer 3

\section{Resumo}

Consonante com o processo de institucionalização da avaliação da atenção primária no Brasil, foi desenvolvida a Matriz Avaliativa do Vínculo Longitudinal (MAVIL), instrumental avaliativo simples e conciso. A MAVIL estrutura-se a partir das três dimensões do conceito de vínculo longitudinal: Dimensão 1 - reconhecimento da unidade básica de saúde (UBS) como fonte regular de cuidados, Dimensão 2 - relação interpessoal profissional/paciente e Dimensão 3 - continuidade da Informação. Consiste em um conjunto estruturado de 12 critérios, 20 indicadores e respectivas questões e itens de verificação, submetido a várias etapas de validação. O presente artigo apresenta a validação estatística da aplicação MAVIL em duas UBS de um território de saúde a fim de respaldar as futuras aplicações e averiguar também a capacidade do instrumento para captar diferenças no perfil do vínculo longitudinal entre UBS. O método consistiu nas seguintes definições: desenho da amostra, escolha da métrica, análise descritiva dos dados e teste comparativo de médias, por meio da aplicação do teste de normalidade e de estatística não paramétrica. Como resultados: a amostra por estrato, junto à opção da soma dos pontos dos itens da MAVIL como métrica, possibilitou descrever o perfil do vínculo longitudinal para os três recortes: território e UBS A e B. Para verificar a capacidade da MAVIL em captar diferença no perfil de vínculo longitudinal, testou-se a hipótese da normalidade dos dados a partir do teste de Kolmogorov-Smirnov. Rejeitada a hipótese da normalidade, optou-se pelo teste não paramétrico de Wilcoxon para comparar as UBS. Para Dimensão 1 e Dimensão 2, constatou-se a semelhança de perfil, contudo, para Dimensão 3, houve diferença significativa, atestando a sensibilidade da MAVIL.

Atenção Primária à Saúde; Estudo de Validação; Pesquisa sobre Serviços de Saúde; Gestão em Saúde

\author{
Correspondência \\ E. M. Cunha \\ Rua Bento Lisboa 165, apto. 504, Rio de Janeiro, RJ \\ 22221-010, Brasil. \\ elenice.cunha@fiocruz.br \\ 1 Escola Politécnica de Saúde Joaquim Venâncio, Fundação \\ Oswaldo Cruz, Rio de Janeiro, Brasil. \\ ${ }_{2}^{2}$ Faculdade de Ciências Humanas, Universidade Federal da \\ Grande Dourados, Dourados, Brasil. \\ 3 Escola Nacional de Saúde Pública Sergio Arouca, Fundação \\ Oswaldo Cruz, Rio de Janeiro, Brasil.
}




\section{Introdução}

No intuito de fortalecer a atenção primária à saúde (APS) no Sistema Único de Saúde (SUS), o Ministério da Saúde tem recomendado, ao longo das duas últimas décadas, a avaliação dos serviços de APS como compromisso da gestão em saúde dos municípios. Em decorrência dessa recomendação, orientou-se que, independentemente da adesão aos ciclos do Programa Nacional de Melhoria do Acesso e da Qualidade da Atenção Básica (PMAQ-AB), todos os municípios avaliassem seus serviços de APS periodicamente, com a prerrogativa de escolha do instrumental avaliativo 1,2.

Para a finalidade de institucionalizar a avaliação da APS, a ferramenta Autoavaliação para a Melhoria do Acesso e da Qualidade da Atenção Básica - (AMAQ) 3 e o Instrumento de Avaliação da Atenção Primária (Primary Care Assessment Tool - PCATool) 4 foram recomendados pelo Ministério da Saúde. A primeira ferramenta estrutura-se a partir de duas unidades de análise: gestão e equipe de saúde, as quais se desdobram em dimensões, subdimensões e itens de verificação, que devem ser objetos de apreciação por parte das equipes. O PCATool contempla os quatro atributos essenciais identificados por Starfield 5 como critérios de qualidade na APS: acesso ao primeiro contato, longitudinalidade, integralidade e coordenação; e, em algumas versões, também os atributos derivados: orientação familiar e comunitária e a competência cultural 6 . Esses atributos se desdobram em questões a serem averiguadas por diferentes fontes. Ambos os instrumentos apresentam número expressivo de questões e tratamento dos dados em várias etapas.

Com o propósito de disponibilizar um instrumental simples e conciso como opção para a avaliação da APS nos municípios e territórios de saúde, uma equipe de pesquisa sediada na Escola Politécnica de Saúde Joaquim Venâncio, Fundação Oswaldo Cruz (EPSJV/Fiocruz) desenvolveu uma metodologia denominada Matriz Avaliativa do Vínculo Longitudinal (MAVIL). Por se tratar de instrumental original em seu conjunto e ao considerar a possibilidade da utilização sistemática por gestores dos diferentes municípios do país, além do aproveitamento para estudos acadêmicos 7 , reconheceu-se, para a MAVIL, a pertinência da realização de etapas da validação, como previsto na literatura sobre o tema 8,9 .

Na primeira fase da pesquisa, foi contemplada a validação de construto, conteúdo e aparência, legitimando a MAVIL em relação à robustez e à representatividade de seus itens ${ }^{10}$. Esse processo teve continuidade com a aplicação da matriz em um território de saúde, completando a validação da aparência e verificando a aplicabilidade e a capacidade do instrumental em medir o vínculo longitudinal.

O presente artigo apresenta o processo de validação da MAVIL que, nesta etapa, tem por objetivos: (1) descrever o tratamento estatístico dos dados obtidos com a aplicação da MAVIL nas duas unidades básicas de saúde (UBS) de um território de saúde e (2) realizar análises estatísticas comparativas a fim de averiguar a sensibilidade do instrumento para captar diferenças no vínculo. Essas análises estatísticas permitirão afirmar a validade dos resultados e respaldarão as futuras aplicações da MAVIL.

Vale ressaltar que iniciativas governamentais, desde 2017, promoveram alterações no arcabouço legal que rege a APS no SUS. De início, houve a flexibilização de alguns componentes da Estratégia Saúde da Família (ESF) previstos na revisão da Politica Nacional de Atenção Básica (PNAB) 11 e, em fins de 2019, a modificação dos critérios de repasses federais para a APS pela Portaria Ministerial no 2.979/2019 12. Essa Portaria, que institui o Programa Previne Brasil, extinguiu o piso per capita e os incentivos financeiros previstos no PMAQ-AB, enfraquecendo o processo avaliativo da APS em curso no país. Contudo, a avaliação de desempenho foi mantida como um dos critérios de repasse, com a possibilidade de os municípios adotarem instrumentos previamente validados para avaliar seus serviços de APS 13. Assim, o desenvolvimento da MAVIL, que vem se afirmando como referência para outros estudos 7,14, poderá se constituir em mais uma opção para que os municípios deem continuidade ao processo de institucionalização da avaliação da APS no SUS. 


\section{Procedimentos metodológicos}

A MAVIL pauta-se no conceito de vínculo longitudinal, compreendido como "relação duradoura entre paciente e profissionais da equipe da APS, que se traduz na utilização da UBS como fonte regular de cuidados ao longo do tempo para os vários episódios de doença e para os cuidados preventivos" 15 (p. 66-7). A construção do referido conceito apoiou-se em revisão de literatura internacional e nacional utilizando os termos "longitudinality" e "continuity of care" 15 e também nos pressupostos de Quivy \& Van Campenhoudt 16 (p. 111) que afirmam que "Construir um conceito consiste primeiro em determinar as dimensões que o constituem (...) e, em seguida, precisar os indicadores graças aos quais as dimensões poderão ser medidas".

A partir dessas bases teóricas e metodológicas, estabeleceu-se que três dimensões compõem o conceito: Dimensão 1 - reconhecimento da equipe de saúde e da UBS como fonte regular de cuidados; Dimensão 2 - relação interpessoal profissional/paciente e Dimensão 3 - continuidade da Informação. Em seguida, foram identificados critérios representativos dessas dimensões. Para expressar esses critérios, elegeram-se indicadores a partir de revisão da produção científica nacional e em acordo aos princípios e diretrizes previstos para a APS no SUS 17. Em continuidade, definiram-se as questões para mensurar os indicadores, sendo que algumas foram elaboradas pela equipe da pesquisa 10,18 , e outras tiveram, por referência, instrumentos existentes, dentre eles, o PCATool.

Sustentada pelo conceito de vínculo longitudinal em suas três dimensões e após validação de construto, conteúdo e aparência 10, a MAVIL estruturou-se em um conjunto de 12 critérios, vinte indicadores e respectivas questões e itens de verificação, dispostos em dois instrumentos de coleta de dados: um questionário com vinte questões referentes aos indicadores das Dimensões 1 e 2, para entrevista com os pacientes, e um formulário com 23 itens de verificação, referentes aos indicadores da Dimensão 3, para revisão do prontuário do paciente entrevistado (Quadros 1 e 2).

Na perspectiva de que a MAVIL seja utilizada para avaliar a APS no SUS, tomou-se como premissa que a primeira aplicação empírica da MAVIL deveria fornecer as bases estatísticas que sustentarão as demais fases da pesquisa. Para tal, tornou-se necessário determinar os limites estatísticos sobre os quais se garantirá a ampla aplicação do método.

Para a primeira aplicação da MAVIL, definiram-se como critérios que o território tivesse cobertura pelos serviços de APS superior a 75\%, e equipes de APS implantadas há mais de dois anos, pressupondo que a presença de tais características é condição para o estabelecimento do vínculo. Para a seleção dos pacientes, foram estabelecidos, como condição traçadora ${ }^{19}$, pacientes com hipertensão arterial primária e/ou diabetes mellitus, bem como os seguintes itens: idade superior a dezoito anos e possuir cadastro na UBS há mais de dois anos. O projeto foi aprovado pelo Comitê de Ética em Pesquisa da EPSJV/Fiocruz sob o no 577621.

O território do estudo foi escolhido não só por conta da cobertura de 100\% pela Estratégia Saúde da Família (ESF) 20, mas também pelo histórico de parcerias com a instituição proponente da pesquisa para realização de estudos. Pertence a uma região caracterizada pelo baixo Índice de Desenvolvimento Humano (IDH) e alto índice de violência urbana. Possuía, em 2016, quando foi realizado o campo da pesquisa, cerca de 45 mil habitantes. As duas UBS do território, aqui identificadas como UBS A e UBS B, possuíam, respectivamente, sete e seis equipes de Saúde da Família e encontravam-se gerenciadas pela mesma Organização Social (OS) com regência em contrato único celebrado entre o gestor municipal e a respectiva OS 21. Por conseguinte, as duas UBS possuíam o mesmo regime de contratação dos profissionais e regras salariais, condições de trabalho similares, além de uma única plataforma de prontuário eletrônico 22 . Em vista desse contexto, a expectativa era não encontrar diferenças de vínculo entre as duas UBS.

O questionário foi aplicado em ambas as UBS entre abril e maio de 2016 em oportunidades e locais de concentração de pacientes com a condição traçadora do estudo, tais como: no dia da campanha de vacinação contra a gripe, nas salas de espera para realização de consulta, antes e após a realização de grupos para pacientes hipertensos e diabéticos e na "academia carioca", que é um local com equipamentos de ginástica onde um profissional de Educação Física conduz e supervisiona exercícios para grupos com necessidades específicas, inclusive para os com hipertensão e/ou diabetes mellitus. O tempo médio de aplicação de cada questionário, extraindo a apresentação e a assinatura do Termo de Consentimento Livre e Esclarecido (TCLE), foi de cerca de cinco minutos. Nos meses de agosto e setembro do mesmo ano, foi realizada a revisão dos prontuários dos pacientes entrevistados. 


\section{A estruturação da MAVIL}

A MAVIL estrutura-se a partir das três dimensões que compõem o conceito de vínculo longitudinal, como pode ser visualizado no Quadro 1. A Dimensão 1 está representada por um único critério: “identificação da UBS/equipe de APS como fonte regular de cuidado”. Esse critério, por sua vez, é medido a partir de três indicadores: (1) identificação da UBS como principal serviço para tratamento de rotina, (2) e também para demanda espontânea e (3) satisfação com o atendimento. Quatro questões, presentes no questionário do usuário, possibilitam a averiguação do alcance dessa dimensão (Quadro 2).

\section{Quadro 1}

Dimensões da Matriz Avaliativa do Vínculo Longitudinal (MAVIL) e respectivos critérios, indicadores, número de questões e total de pontos possíveis por critério e por dimensão.

\begin{tabular}{|c|c|c|c|}
\hline $\begin{array}{l}\text { CRITÉRIOS (C) DE CADA } \\
\text { DIMENSÃO (D) }\end{array}$ & INDICADORES DE CADA CRITÉRIO & $\begin{array}{l}\text { NÚMERO DE QUESTÕES } \\
\text { POR CRITÉRIO E TOTAL POR } \\
\text { DIMENSÃO }\end{array}$ & $\begin{array}{l}\text { TOTAL DE PONTOS } \\
\text { POR DIMENSÃO E } \\
\text { POR INDICADOR } \\
\end{array}$ \\
\hline \multicolumn{2}{|c|}{$\begin{array}{l}\text { DIMENSÃO 1: identificação da equipe de APS/UBS como fonte regular de } \\
\text { cuidados (D1) }\end{array}$} & 4 & 35 \\
\hline \multirow{3}{*}{$\begin{array}{l}\text { C1 - Identificação da UBS } \\
\text { como referência para o } \\
\text { cuidado }\end{array}$} & $\begin{array}{l}\text { Identificação da equipe de APS/UBS como serviço } \\
\text { de busca regular. }\end{array}$ & 2 & 20 \\
\hline & $\begin{array}{l}\text { Procura da equipe de APS/UBS para problemas } \\
\text { de saúde, excetuando o tratamento de rotina. }\end{array}$ & 1 & 10 \\
\hline & $\begin{array}{l}\text { Mudaria de equipe de APS/UBS se tivesse esta } \\
\text { opção. }\end{array}$ & 1 & 5 \\
\hline \multicolumn{2}{|c|}{ DIMENSÃO 2: relação interpessoal profissional/paciente (D2) } & 16 & 150 \\
\hline \multirow{3}{*}{$\begin{array}{l}\text { C1 - Existência de médico e } \\
\text { de enfermeiro de referência } \\
\text { para o atendimento de rotina }\end{array}$} & $\begin{array}{l}\text { Existência de um médico de referência para o } \\
\text { atendimento de rotina. }\end{array}$ & 2 & 15 \\
\hline & $\begin{array}{l}\text { Existência de um enfermeiro de referência para o } \\
\text { atendimento de rotina. }\end{array}$ & 2 & 15 \\
\hline & $\begin{array}{c}\text { Reconhecimento de outros profissionais da } \\
\text { equipe de saúde como referência para o } \\
\text { atendimento. }\end{array}$ & 2 & 10 \\
\hline \multirow{4}{*}{$\begin{array}{l}\text { C2 - Confiança nos } \\
\text { profissionais de referência }\end{array}$} & Confiança no médico de referência. & 2 & 30 \\
\hline & $\begin{array}{l}\text { Interesse do médico pelos problemas do } \\
\text { paciente. }\end{array}$ & 1 & 10 \\
\hline & Confiança no enfermeiro de referência. & 1 & 10 \\
\hline & $\begin{array}{l}\text { Interesse do enfermeiro pelos problemas do } \\
\text { paciente. }\end{array}$ & 1 & 10 \\
\hline \multirow{2}{*}{$\begin{array}{l}\text { C3 - Conhecimento dos } \\
\text { aspectos psicossociais do } \\
\text { paciente pelos profissionais } \\
\text { de referência }\end{array}$} & $\begin{array}{l}\text { Conhecimento do médico sobre aspectos } \\
\text { biopsicossociais do paciente. }\end{array}$ & 1 & 10 \\
\hline & $\begin{array}{l}\text { Conhecimento do enfermeiro sobre aspectos } \\
\text { biopsicossociais do paciente. }\end{array}$ & 1 & 10 \\
\hline $\begin{array}{l}\text { C4 - Tempo suficiente para o } \\
\text { atendimento }\end{array}$ & $\begin{array}{l}\text { Tempo para esclarecimentos durante as } \\
\text { consultas. }\end{array}$ & 1 & 10 \\
\hline \multirow{2}{*}{$\begin{array}{l}\text { C5 - Orientações de forma } \\
\text { que o paciente compreenda }\end{array}$} & Compreensão da fala do médico. & 1 & 10 \\
\hline & Compreensão da fala do enfermeiro. & 1 & 10 \\
\hline
\end{tabular}

(continua) 


\begin{tabular}{|c|c|c|c|}
\hline $\begin{array}{l}\text { CRITÉRIOS (C) DE CADA } \\
\text { DIMENSÃO (D) }\end{array}$ & INDICADORES DE CADA CRITÉRIO & $\begin{array}{l}\text { NÚMERO DE QUESTÕES } \\
\text { POR CRITÉRIO E TOTAL POR } \\
\text { DIMENSÃO }\end{array}$ & $\begin{array}{l}\text { TOTAL DE PONTOS } \\
\text { POR DIMENSÃO E POR } \\
\text { INDICADOR }\end{array}$ \\
\hline \multicolumn{2}{|c|}{ DIMENSÃO 3: continuidade da informação (D3) } & 23 & 130 \\
\hline $\begin{array}{l}\text { C1 - Adequação do } \\
\text { quantitativo de consultas }\end{array}$ & Registro de número de consultas. & 2 & 26 \\
\hline $\begin{array}{l}\text { C2 - Organização geral do } \\
\text { prontuário }\end{array}$ & Clareza e organização dos registros. & 2 & 16 \\
\hline $\begin{array}{l}\text { C3 - Registro de dados do } \\
\text { exame físico }\end{array}$ & $\begin{array}{l}\text { Prontuários com registro adequado do exame } \\
\text { físico. }\end{array}$ & 4 & 24 \\
\hline $\begin{array}{l}\text { C4 - Registro dos exames } \\
\text { complementares }\end{array}$ & Registro dos exames laboratoriais. & 8 & 16 \\
\hline $\begin{array}{l}\text { C5 - Registro da conduta } \\
\text { prescritiva }\end{array}$ & Registro da conduta terapêutica. & 5 & 40 \\
\hline $\begin{array}{l}\text { C6 - Registro de aspectos } \\
\text { psicossociais }\end{array}$ & Registros de aspectos psicossociais. & 2 & 8 \\
\hline
\end{tabular}

APS: atenção primária à saúde; UBS: unidades básicas de saúde.

Nota: para a revisão dos prontuários, os dois anos objeto da revisão foram subdivididos em quatro períodos de seis meses, posto que os protocolos do Ministério da Saúde preconizam, ao menos, uma consulta por semestre.

A Dimensão 2 - "relação interpessoal profissional de saúde/paciente" - é representada por cinco critérios, a saber: (1) reconhecimento dos profissionais médico e enfermeiro de referência, (2) confiança nos profissionais de referência, (3) reconhecimento de aspectos biopsicossociais do paciente por parte dos profissionais de saúde, (4) tempo para esclarecimento de dúvidas durante as consultas e (5) compreensão da fala dos profissionais (Quadro 1). A mensuração desses critérios ocorre a partir de 12 indicadores, averiguados por 16 questões contempladas no questionário do usuário (Quadro 2).

A Dimensão 3 - "continuidade da informação" - refere-se à organização do prontuário e ao registro das preconizações para as condições traçadoras do estudo e encontra-se representada por seis critérios, a saber: (1) adequação do quantitativo de consultas, (2) organização geral do prontuário, (3) registro de dados do exame físico, (4) registro dos exames complementares, (5) registro da conduta prescritiva e (6) registro de aspectos psicossociais (Quadro 1). Cada um dos seis critérios da Dimensão 3 possui um único indicador, averiguável em seu conjunto por 23 itens presentes no formulário de revisão do prontuário. O Quadro 1 sintetiza a estruturação da MAVIL e apresenta a pontuação por dimensão e indicador. O Quadro 2 apresenta questões e itens da MAVIL e a síntese da regra de pontuação.

\section{Desenho da amostra}

Para a presente pesquisa, foi aplicado o modelo de amostragem estratificada semelhante à amostragem da Pesquisa Nacional de Saúde (PNS) 23, aplicando o seu conceito para as regiões geográficas do território estudado. Cada unidade do território foi compreendida como um estrato. Esse desenho amostral permite ter representatividade, proporcional ou ótima, quando se leva em conta a variabilidade da amostra além do peso de cada estrato.

O cálculo da amostra foi realizado usando amostra estratificada proporcional, dado que foi maximizada a variância da distribuição Bernoulli 24,25,26,27, usando uma função de custo linear e considerando o custo fixo. É uma fórmula genérica aplicável a qualquer território como exigido pela MAVIL. 
Quadro 2

Questões e itens e respectiva regra de pontuação por critério e dimensão da Matriz Avaliativa do Vínculo Longitudinal (MAVIL).

\begin{tabular}{|c|c|c|c|}
\hline $\begin{array}{l}\text { DIMENSÃO/CRITÉRIO/ } \\
\text { INDICADOR (D/C/I) }\end{array}$ & QUESTÕES & REGRA DE PONTUAÇÃO & PONTOS \\
\hline \multicolumn{3}{|c|}{$\begin{array}{l}\text { DIMENSÃO 1: identificação da equipe de APS/UBS como fonte regular de cuidados } \\
1 \text { critério, } 3 \text { indicadores e } 4 \text { questões - fonte: entrevista com o paciente. }\end{array}$} & 35 \\
\hline \multirow[t]{2}{*}{$\mathrm{D} 1 / \mathrm{C} 1 / \mathrm{I} .1$} & $\begin{array}{l}\text { Qual unidade de saúde o Sr. costuma ir quando } \\
\text { precisa de atendimento de saúde? }\end{array}$ & $\begin{array}{l}\text { Se marcar opção "a" “unidade básica pesquisada": } \\
10 \text { pontos; outra opção: não pontua; se marcar a } \\
\text { "a" junto com outra opção, perde } 5 \text { pontos. }\end{array}$ & 0,5 ou 10 \\
\hline & $\begin{array}{l}\text { Os profissionais da equipe da UBS vão ao seu } \\
\text { domicílio para saber sobre sua saúde? }\end{array}$ & Opções: Sim - 10 pontos; Não - 0 & 0 ou 10 \\
\hline $\mathrm{D} 1 / \mathrm{C} 1 / \mathrm{l} .2$ & $\begin{array}{l}\text { Por quais motivos costuma vir a este posto de } \\
\text { saúde? }\end{array}$ & $\begin{array}{l}\text { Se marcar opção "a" (consulta/doença crônica): } \\
8 \text { pontos; outra opção: 0; se marcar a "a", junto } \\
\text { com outra opção, acrescenta } 2 \text { pontos. }\end{array}$ & 0 a 10 \\
\hline D1/C1/l.3 & $\begin{array}{l}\text { Se tivesse oportunidade, mudaria de unidade ou } \\
\text { de equipe? }\end{array}$ & $\begin{array}{l}\text { Opção "Não": } 5 \text { pontos - espaço aberto para } \\
\text { justificativa da resposta. }\end{array}$ & 0 ou 5 \\
\hline \multicolumn{3}{|c|}{$\begin{array}{l}\text { DIMENSÃo 2: relação interpessoal profissional/paciente } \\
5 \text { critérios, } 12 \text { indicadores e } 16 \text { questões - fonte: entrevista com o paciente. }\end{array}$} & 150 \\
\hline \multirow[t]{2}{*}{$\mathrm{D} 2 / \mathrm{C} 1 / \mathrm{I} .1$} & $\begin{array}{l}\text { O Sr. tem um médico dessa unidade de saúde que o } \\
\text { atende regularmente? }\end{array}$ & Opção “Sim": 10 pontos. & 0 ou 10 \\
\hline & Caso positivo, qual o nome desse médico? & Se souber o nome: 5 pontos. & 0 ou 5 \\
\hline \multirow[t]{2}{*}{$\mathrm{D} 2 / \mathrm{C} 1 / \mathrm{l} .2$} & $\begin{array}{l}\text { O Sr. tem enfermeiro(a) dessa unidade de saúde que } \\
\text { o atende regularmente? }\end{array}$ & Opção "Sim": 10 pontos. & 0 ou 10 \\
\hline & Caso positivo, qual o nome desse enfermeiro? & Se souber o nome: 5 pontos. & 0 ou 5 \\
\hline \multirow[t]{2}{*}{$\mathrm{D} 2 / \mathrm{C} 1 / \mathrm{l} .3$} & $\begin{array}{l}\text { Quais outros profissionais o Sr. recorre quando } \\
\text { precisa de atendimento nessa unidade? }\end{array}$ & Opção "Sim": 5 pontos. & 0 ou 5 \\
\hline & Caso positivo, qual(is) o(s) nome(s)? & Se souber o nome: 5 pontos. & 0 ou 5 \\
\hline \multirow[t]{2}{*}{$\mathrm{D} 2 / \mathrm{C} 2 / \mathrm{l} .4$} & O Sr. confia no médico que o atende regularmente? & $\begin{array}{l}\text { a) Sempre: } 20 \\
\text { b) Quase sempre: } 15 \\
\text { c) Poucas vezes: } 1 \\
\text { d) Nunca: } 0\end{array}$ & 0 a 20 \\
\hline & $\begin{array}{l}\text { O Sr. se sente à vontade para tirar dúvidas durante } \\
\qquad \text { a consulta? }\end{array}$ & $\begin{array}{l}\text { a) Sempre: } 10 \\
\text { b) Quase sempre: } 8 \\
\text { c) Poucas vezes: } 1 \\
\text { d) Nunca: } 0\end{array}$ & 0 a 10 \\
\hline $\mathrm{D} 2 / \mathrm{C} 2 / \mathrm{I} .5$ & $\begin{array}{c}\text { O médico que o atende regularmente se interessa } \\
\text { pelos seus problemas de saúde? }\end{array}$ & $\begin{array}{l}\text { a) Sempre: } 10 \\
\text { b) Quase sempre: } 8 \\
\text { c) Poucas vezes: } 1 \\
\text { d) Nunca: } 0\end{array}$ & 0 a 10 \\
\hline $\mathrm{D} 2 / \mathrm{C} 2 / \mathrm{I} .6$ & $\begin{array}{c}\text { O Sr. confia no enfermeiro que o atende } \\
\text { regularmente? }\end{array}$ & $\begin{array}{l}\text { a) Sempre: } 10 \\
\text { b) Quase sempre: } 8 \\
\text { c) Poucas vezes: } 1 \\
\text { d) Nunca: } 0\end{array}$ & 0 a 10 \\
\hline $\mathrm{D} 2 / \mathrm{C} 2 / \mathrm{I} .7$ & $\begin{array}{c}\text { O enfermeiro que o atende regularmente se } \\
\text { interessa por seus problemas de saúde? }\end{array}$ & $\begin{array}{l}\text { a) Sempre: } 10 \\
\text { b) Quase sempre: } 8 \\
\text { c) Poucas vezes: } 1 \\
\text { d) Nunca: } 0\end{array}$ & 0 a 10 \\
\hline
\end{tabular}

(continua) 


\begin{tabular}{|c|c|c|c|}
\hline $\begin{array}{l}\text { DIMENSÃO/CRITÉRIO/ } \\
\text { INDICADOR (D/C/I) }\end{array}$ & QUESTÕES & REGRA DE PONTUAÇÃO & PONTOS \\
\hline $\mathrm{D} 2 / \mathrm{C} 3 / \mathrm{l} .8$ & $\begin{array}{c}\text { Considera que o médico que o atende nesta unidade } \\
\text { sabe o que precisa saber sobre sua vida para tratá- } \\
\text { lo de forma correta? }\end{array}$ & $\begin{array}{l}\text { a) Tudo: } 10 \\
\text { b) Bastante: } 8 \\
\text { c) Pouco: } 6 \\
\text { d) Nada: } 0\end{array}$ & 0 a 10 \\
\hline $\mathrm{D} 2 / \mathrm{C} 3 / \mathrm{I} .9$ & $\begin{array}{c}\text { Considera que o enfermeiro que o atende nesta } \\
\text { unidade sabe o que precisa saber sobre sua vida } \\
\text { para tratá-lo de forma correta? }\end{array}$ & $\begin{array}{l}\text { a) Tudo: } 10 \\
\text { b) Bastante: } 8 \\
\text { c) Pouco: } 6 \\
\text { d) Nada: } 0\end{array}$ & 0 a 10 \\
\hline D2/C4/I.10 & $\begin{array}{c}\text { Durante a consulta, o Sr. tem tempo suficiente para } \\
\text { tirar dúvidas? }\end{array}$ & $\begin{array}{l}\text { a) Sempre: } 10 \\
\text { b) Quase sempre: } 8 \\
\text { c) Poucas vezes: } 4 \\
\text { d) Nunca: } 0\end{array}$ & 0 a 10 \\
\hline D2/C5 /l.11 & $\begin{array}{l}\text { O Sr. compreende as orientações do médico durante } \\
\qquad \text { a consulta? }\end{array}$ & $\begin{array}{l}\text { a) Sempre: } 10 \\
\text { b) Quase sempre: } 8 \\
\text { c) Poucas vezes: } 1 \\
\text { d) Nunca: } 0\end{array}$ & 0 a 10 \\
\hline D2/C5/I.12 & $\begin{array}{c}\text { O Sr. compreende as orientações do enfermeiro } \\
\text { durante os atendimentos? }\end{array}$ & $\begin{array}{l}\text { a) Sempre: } 10 \\
\text { b) Quase sempre: } 8 \\
\text { c) Poucas vezes: } 1 \\
\text { d) Nunca: } 0\end{array}$ & 0 a 10 \\
\hline \multicolumn{3}{|c|}{$\begin{array}{l}\text { DIMENSÃo 3: continuidade da informação } \\
6 \text { critérios, } 6 \text { indicadores e } 23 \text { itens de verificação - fonte: prontuário do paciente. }\end{array}$} & 130 \\
\hline \multirow[t]{2}{*}{ D3/C1/l.1 } & 4 ou mais consultas no período. & 10 & \multirow[t]{2}{*}{0 a 26} \\
\hline & Consulta com médico e com enfermeiro no período? & 2 por período $=16$ & \\
\hline \multirow[t]{2}{*}{$\mathrm{D} 3 / \mathrm{C} 2 / 1.2$} & Os registros estão legíveis? & 2 por período $=8$ & \multirow[t]{2}{*}{0 a 16} \\
\hline & $\begin{array}{l}\text { Os registros estão organizados em ordem } \\
\text { cronológica? }\end{array}$ & 2 por período $=8$ & \\
\hline \multirow[t]{2}{*}{$\mathrm{D} 3 / \mathrm{C} 3 / 1.3$} & Pressão arterial. & 3 por período $=12$ & \multirow[t]{2}{*}{0 a 24} \\
\hline & $\begin{array}{l}\text { Peso, IMC, perímetro abdominal } \\
\text { (3 itens). }\end{array}$ & $\begin{array}{l}\text { Período } 1 \text { e/ou } 2 \text { = } 2 \text { por item }=6 \\
\text { Períodos } 3 \text { e/ou } 4=2 \text { por item }=6\end{array}$ & \\
\hline D3/C4/I.4 & $\begin{array}{c}\text { Glicemia de jejum, hemoglobina glicosilada, } \\
\text { triglicerídeos, colesterol total, HDL, creatinina, } \\
\text { EAS, microalbuminúria (8 itens). }\end{array}$ & 0,5 por item multiplicado pelo período $=0,5 \times 8 \times 4$ & 0 a 16 \\
\hline \multirow[t]{5}{*}{$\mathrm{D} 3 / \mathrm{C} 5 / \mathrm{I} .5$} & $\begin{array}{l}\text { Se hipertenso, classificação - se somente } \\
\text { diabético também recebe pontuação. }\end{array}$ & Registro em qualquer período $=10$ & \multirow[t]{5}{*}{0 a 40} \\
\hline & Registro do risco cardíaco. & Registro em qualquer período $=10$ & \\
\hline & Registro do medicamento. & 2 por período $=8$ & \\
\hline & $\begin{array}{l}\text { Orientações - medidas de promoção e } \\
\text { prevenção. }\end{array}$ & Registro em qualquer período $=4$ & \\
\hline & Retorno dos encaminhamentos. & 2 por período $=8$ & \\
\hline \multirow[t]{2}{*}{ D3/C6/I.6 } & Há registros de aspectos psicossociais? & Registro em qualquer período = 4 & \multirow[t]{2}{*}{0 a 8} \\
\hline & Há registro da escolaridade? & Registro em qualquer período $=4$ & \\
\hline
\end{tabular}

APS: atenção primária à saúde; IMC: índice de massa corporal; UBS: unidades básicas de saúde.

Nota: para a revisão dos prontuários, os dois anos objeto da revisão foram subdivididos em quatro períodos de seis meses, posto que os protocolos do Ministério da Saúde preconizam, ao menos, uma consulta por semestre. 
As informações a priori, necessárias para fazer a amostra estratificada, foram obtidas no cadastro de usuários das unidades: total populacional, sexo, unidade de saúde e morbidade. Conforme definido nesse instrumento, como condição traçadora, foram contados todos os que possuíam dois ou mais anos de prontuário nas unidades de saúde e com as morbidades hipertensão e/ou diabetes. Nesse caso, estabelecendo o total de 5.294 pacientes, sendo 3.380 na UBS A e 1.914 na UBS B.

Optou-se por uma amostra sem caso-controle, mas que permitisse verificar o perfil do vínculo no grupo estudado. Então, a amostra foi calculada considerando as três variáveis para estratificação e eliminando variáveis até que o tamanho da amostra fosse compatível com o orçamento e o cronograma da pesquisa. Ficou viável a amostra calculada considerando a estratificação apenas pelas unidades de saúde. Como o universo de pacientes com a condição traçadora nas respectivas UBS era de 5.294 pacientes, o tamanho da amostra necessário para o nível de $90 \%$ de significância foi de 186 pacientes, sendo 94, para a UBS A, e de 92, para a UBS B.

Por segurança e com base na experiência de campo dos pesquisadores, prevendo-se alguma perda, foram entrevistados 202 pacientes. O limite para o número de entrevistas foi o prazo do cronograma da pesquisa. Do total de entrevistados, somente um (1) foi rejeitado por não haver prontuário na unidade. Consequentemente, a amostra final foi de 201 pacientes entrevistados, que também tiveram seus prontuários revisados: 102 da UBS A e 99 da UBS B.

A partir desse contexto, os dados empíricos foram analisados de acordo com as seguintes etapas: (i) escolha da estatística; (ii) análise descritiva; (iii) teste de normalidade; (iv) estatística não paramétrica; e (v) teste comparativo de médias.

\section{Resultados}

\section{Escolha da estatística}

A análise estatística exige a definição de uma métrica que possa ser estudada quantitativamente. No caso do presente estudo, essa métrica representará a medida de vínculo que se pretende avaliar. Por atender aos requisitos de simplicidade de aplicação, facilidade de compreensão e possibilidade de comparação de métricas alternativas que possam ser aplicadas futuramente, a escolha foi pela soma direta dos pontos de cada item da matriz.

A partir da opção de soma direta, em que o conjunto de indicadores da Dimensão 1 soma 35 pontos; da Dimensão 2 soma 150 pontos; e da Dimensão 3 soma 130 pontos, a MAVIL, em seu conjunto de itens, totaliza 315 pontos para cada paciente sujeito da pesquisa, sendo esse total a expressão do maior vínculo possível (Quadro 1). Considerando que a amostra foi de 201 pacientes, o máximo de pontos possíveis do território seria de 63.315 pontos, sendo que a UBS A, com amostra de 102 pacientes, poderia alcançar 32.130; e a UBS B, cuja amostra foi de 99 pacientes, poderia alcançar 31.185 pontos (Tabela 1).

Desse modo, as variáveis quantitativas definidas por essa métrica são os resultantes da soma dos pontos de todos os itens do questionário do paciente e do formulário de revisão de prontuários. Exemplificando: para o critério 1, a soma dos pontos de todos os itens do questionário do referido critério constitui-se em uma variável. Então, considerando que a MAVIL possui 12 critérios, tem-se um conjunto de 12 variáveis. Acrescido do total das dimensões, que são três, e ainda a totalidade da MAVIL, são obtidas 16 variáveis. Essas 16 variáveis foram analisadas em três recortes diferentes: Território, UBS A e UBS B.

\section{Análise descritiva para o território e para as unidades}

A aplicação da MAVIL no território alcançou 32.460 pontos, o equivalente a 51,3\% dos pontos possíveis. Para a Dimensão 1, a pontuação alcançada foi de 5.203, de um total possível de 7.035 pontos, o que correspondeu a 74\% desse total, sendo obtidos a média de 25,9 e o desvio padrão de 7,48, o que gerou um coeficiente de variação de 0,29. Para a Dimensão 2, a pontuação alcançada foi de 15.850, de uma soma máxima possível de 30.150, o que correspondeu a 52,6\% do total, sendo obtidos a média de 78,9 e o desvio padrão de 44,95, o que apontou um coeficiente de variação de 0,57 . Para a Dimensão 
Tabela 1

Total das unidades básicas de saúde (UBS) e do território por dimensões, por estatísticas descritivas.

\begin{tabular}{|c|c|c|c|c|}
\hline Local & Total MAVIL & Dimensão 1 & Dimensão 2 & Dimensão 3 \\
\hline \multicolumn{5}{|l|}{ UBS A } \\
\hline N válido & 102 & 102 & 102 & 102 \\
\hline Média & 158,2 & 25,2 & 80,3 & 52,8 \\
\hline Mediana & 166,8 & 25,0 & 80,0 & 57,3 \\
\hline Moda & 97,0 & 33,0 & 0,0 & 16,0 \\
\hline Desvio padrão & 57,0 & 7,5 & 47,7 & 20,4 \\
\hline Amplitude & 232,5 & 35,0 & 144,0 & 75,0 \\
\hline Mínimo & 26,0 & 0,0 & 0,0 & 16,0 \\
\hline Máximo & 258,5 & 35,0 & 144,0 & 75,0 \\
\hline Soma & $16.136,5$ & $2.567,0$ & $8.187,0$ & $5.382,5$ \\
\hline Máximo possível & $32.130,0$ & $3.570,0$ & $15.300,0$ & $13.260,0$ \\
\hline Percentual & 50,22 & 71,90 & 53,51 & 40,59 \\
\hline Coeficiente de variação & 0,36 & 0,30 & 0,59 & 0,39 \\
\hline Percentil 25 & 110,0 & 20,0 & 53,0 & 34,0 \\
\hline Percentil 50 & 166,8 & 25,0 & 80,0 & 57,3 \\
\hline Percentil 75 & 203,9 & 33,0 & 127,3 & 68,3 \\
\hline \multicolumn{5}{|l|}{ UBS B } \\
\hline N válido & 99 & 99 & 99 & 99 \\
\hline Média & 164,9 & 26,6 & 77,4 & 60,9 \\
\hline Mediana & 166,0 & 28,0 & 80,0 & 63,0 \\
\hline Moda & 149,0 & 35,0 & 4,0 & 63,0 \\
\hline Desvio padrão & 51,7 & 7,4 & 42,2 & 17,5 \\
\hline Amplitude & 224,5 & 30,0 & 148,0 & 76,0 \\
\hline Mínimo & 33,0 & 5,0 & 0,0 & 16,0 \\
\hline Máximo & 257,5 & 35,0 & 148,0 & 92,0 \\
\hline Soma & $16.323,5$ & $2.636,0$ & $7.663,0$ & $6.024,5$ \\
\hline Máximo possível & $31.185,0$ & $3.465,0$ & $14.850,0$ & $12.870,0$ \\
\hline Percentual & 52,34 & 76,08 & 51,60 & 46,81 \\
\hline Coeficiente de variação & 0,31 & 0,28 & 0,54 & 0,29 \\
\hline Percentil 25 & 135,0 & 23,0 & 58,0 & 52,0 \\
\hline Percentil 50 & 166,0 & 28,0 & 80,0 & 63,0 \\
\hline Percentil 75 & 204,5 & 33,0 & 113,0 & 73,5 \\
\hline \multicolumn{5}{|l|}{ Total do território } \\
\hline N válido & 201 & 201 & 201 & 201 \\
\hline Média & 161,0 & 25,9 & 78,9 & 56,8 \\
\hline Mediana & 166,0 & 27,0 & 80,0 & 60,5 \\
\hline Moda & 149,0 & 35,0 & 4,0 & 16,0 \\
\hline Desvio padrão & 54,4 & 7,5 & 45,0 & 19,4 \\
\hline Amplitude & 232,5 & 35,0 & 148,0 & 76,0 \\
\hline Mínimo & 26,0 & 0,0 & 0,0 & 16,0 \\
\hline Máximo & 258,5 & 35,0 & 148,0 & 92,0 \\
\hline Soma & $32.450,0$ & $5.203,0$ & $15.850,0$ & $11.407,0$ \\
\hline Máximo possível & $63.315,0$ & $7.035,0$ & $30.150,0$ & $26.130,0$ \\
\hline Percentual & 51,27 & 73,96 & 52,57 & 43,65 \\
\hline Coeficiente de variação & 0,34 & 0,29 & 0,57 & 0,34 \\
\hline Percentil 25 & 121,5 & 21,0 & 55,5 & 46,8 \\
\hline Percentil 50 & 166,0 & 27,0 & 80,0 & 60,5 \\
\hline Percentil 75 & 204,0 & 33,0 & 121,0 & 70,0 \\
\hline
\end{tabular}

MAVIL: Matriz Avaliativa do Vínculo Longitudinal.

Nota: não houve número faltante. Tabela executada no software SPSS (https://www.ibm.com/). 
3, a pontuação alcançada foi de 11.407 , de um total possível de 26.130 , o que correspondeu a $43,7 \%$ desse total, sendo obtidos a média de 56,75 e o desvio padrão de 19,4 , com coeficiente de variação de 0,34 (Tabela 1).

Com relação à dispersão dos dados, pode-se observar que a Dimensão 1 apresentou os resultados mais coesos, ou seja, os usuários mais parecidos em suas respostas. As Dimensões 2 e 3 apresentaram valores elevados da dispersão, sendo a Dimensão 2 com o maior coeficiente de variação (Tabela 1).

Com relação à análise descritiva das unidades, a partir da métrica utilizada, a UBS A atingiu $50,22 \%$ dos pontos, com variação individual de 26 a 258,5 pontos e mediana de 166,8 ; a UBS B obteve $52,34 \%$ dos pontos com variação de 33 a 257,5 pontos e mediana de 166 (Tabela 1). A UBS B obteve melhor desempenho nas Dimensões 1 e 3, identificação da equipe de APS/UBS como fonte regular de cuidados e continuidade da informação, respectivamente.

No que tange à comparação entre as UBS, em relação à dispersão dos dados, observa-se que a Dimensão 1 apresentou os resultados mais coesos (desvio padrão de 7,5 para UBS A e de 7,4 para UBS B), e a Dimensão 2 apresentou maior dispersão entre as duas unidades, sendo o desvio padrão de 47,7 para UBS A e de 42,2 para a UBS B.

Para a Dimensão 1, identificação da equipe de APS/UBS como fonte regular de cuidados, a UBS A obteve $71,9 \%$ do máximo de pontos possíveis, e a mediana foi de 25 pontos; enquanto a UBS B alcançou $76 \%$ do máximo de pontos possíveis, com mediana de 28 pontos. Na Dimensão 2, relação interpessoal profissional/paciente, as unidades A e B atingiram, respectivamente, 53,5\% e 51,6\% dos pontos possíveis, e a mediana foi igual para ambas (80 pontos). A Dimensão 3 , continuidade da informação, atingiu 40,59\% dos pontos para a UBS A e 46,81\% dos pontos para a UBS B (Tabela 1).

As estatísticas descritivas das duas unidades apresentaram resultados semelhantes, destacando apenas a Dimensão 3, que apresentou maior diferença: a UBS B com mediana de 63 pontos de escore e a UBS A com 57,3 pontos de escore, uma diferença de 5,7 pontos.

Observa-se, na Tabela 2, que o percentual de pontos atingidos dos critérios das Dimensões 1 e 3 na UBS B são maiores que na UBS A. Em relação à Dimensão 2, a UBS B ultrapassa a UBS A nos critérios 2 e 4 (confiança nos profissionais de referência e tempo suficiente para o atendimento, respectivamente).

\section{Teste de normalidade}

Na intenção do uso sistemático da MAVIL, estabelecer um teste que permita aos gestores, ao utilizar esse instrumental avaliativo, afirmar a validade dos resultados da aplicação é condição sine qua non. Nessa etapa da pesquisa, para verificar a capacidade da MAVIL em captar diferença no vínculo estabelecido, fez-se a comparação das duas UBS do território a partir de um teste de hipótese. Para se utilizar um teste de hipótese paramétrico, é preciso testar a normalidade, sendo esse o primeiro passo.

Aplicado o teste de Kolmogorov-Smirnov na amostra para cada uma das dimensões e para o conjunto das dimensões em sua totalidade, o resultado foi a aceitação da normalidade apenas para o total, com valor de $\mathrm{p}=0,1032$, e, para a terceira dimensão, com valor de $\mathrm{p}=0,0589$, sendo essa com o valor crítico muito próximo ao valor de rejeição que é de $5 \%(\alpha=0,05)$. A primeira dimensão e a segunda dimensão rejeitam a hipótese de normalidade, com valor de $\mathrm{p}=0,0011$ e 0,0247, respectivamente.

\section{Estatística não paramétrica}

$\mathrm{O}$ Teste $\mathrm{t}$ não foi realizado porque a amostra não cumpriu o pressuposto da normalidade dos dados para se comparar em duas amostras. Diante desse fato, a opção foi adotar testes de hipótese não paramétricos. Os testes não paramétricos ganham em generalização, sem pressupostos rígidos (como normalidade dos dados ou formato da distribuição), podendo ser aplicados a praticamente todas as bases de dados. Dessa forma, podem ser aplicados para a utilização da MAVIL para a comparação de quaisquer duas UBS ou territórios. 
Tabela 2

Critérios das dimensões da Matriz Avaliativa do Vínculo Longitudinal (MAVIL) por estatísticas descritivas.

\begin{tabular}{|c|c|c|c|c|c|c|c|c|c|c|c|c|}
\hline Critérios & D1/C1 & $\mathrm{D} 2 / \mathrm{C} 1$ & $\mathrm{D} 2$ /C2 & $\mathrm{D} 2 / \mathrm{C} 3$ & $\mathrm{D} 2 / \mathrm{C} 4$ & D2/C5 & D3/C1 & D3/C23 & D3/C3 & D3/C4 & D3/C5 & D3/C6 \\
\hline Pontos & 35 & 40 & 60 & 20 & 10 & 20 & 26 & 16 & 24 & 16 & 40 & 8 \\
\hline \multicolumn{13}{|l|}{ UBS A } \\
\hline N válido & 102 & 102 & 102 & 102 & 102 & 102 & 102 & 102 & 102 & 102 & 102 & 102 \\
\hline Média & 25,2 & 21,9 & 32,8 & 9,7 & 3,4 & 12,5 & 13,1 & 16,0 & 11,6 & 1,5 & 7,1 & 3,5 \\
\hline Mediana & 25,0 & 25,0 & 34,5 & 10,0 & 4,0 & 10,0 & 16,0 & 16,0 & 13,0 & 0,0 & 6,0 & 4,0 \\
\hline Moda & 33,0 & 25,0 & 60,0 & 0,0 & 4,0 & 20,0 & 18,0 & 16,0 & 17,0 & 0,0 & 0,0 & 4,0 \\
\hline Desvio padrão & 7,5 & 12,5 & 23,1 & 6,9 & 2,2 & 7,6 & 8,1 & 0,0 & 6,8 & 2,1 & 6,4 & 1,7 \\
\hline Mínimo & 0,0 & 0,0 & 0,0 & 0,0 & 0,0 & 0,0 & 0,0 & 16,0 & 0,0 & 0,0 & 0,0 & 0,0 \\
\hline Máximo & 35,0 & 40,0 & 60,0 & 20,0 & 8,0 & 20,0 & 24,0 & 16,0 & 22,0 & 8,0 & 27,0 & 6,0 \\
\hline Soma & $2.567,0$ & $2.230,0$ & $3.345,0$ & 990,0 & 344,0 & $1.278,0$ & $1.332,0$ & $1.632,0$ & $1.186,0$ & 152,5 & 722,0 & 358,0 \\
\hline Máximo possível & 3.570 & 4.080 & 6.120 & 2.040 & 1.020 & 2.040 & 2.652 & 1.632 & 2.448 & 1.632 & 4.080 & 816 \\
\hline Percentual & 71,9 & 54,7 & 54,7 & 48,5 & 33,7 & 62,6 & 50,2 & 100,0 & 48,4 & 9,3 & 17,7 & 43,9 \\
\hline Coeficiente de variação & 0,30 & 0,57 & 0,70 & 0,71 & 0,66 & 0,61 & 0,62 & 0,00 & 0,58 & 1,38 & 0,91 & 0,50 \\
\hline Percentil 25 & 20,0 & 13,8 & 13,8 & 2,0 & 3,0 & 9,5 & 4,0 & 16,0 & 7,0 & 0,0 & 2,0 & 4,0 \\
\hline Percentil 50 & 25,0 & 25,0 & 34,5 & 10,0 & 4,0 & 10,0 & 16,2 & 16,0 & 13,0 & 0,0 & 6,0 & 4,0 \\
\hline Percentil 75 & 33,0 & 30,0 & 60,0 & 16,0 & 4,0 & 20,0 & 20,0 & 16,0 & 17,0 & 2,6 & 10,0 & 4,0 \\
\hline \multicolumn{13}{|l|}{ UBS B } \\
\hline N válido & 99 & 99 & 99 & 99 & 99 & 99 & 99 & 99 & 99 & 99 & 99 & 99 \\
\hline Média & 26,6 & 19,7 & 33,9 & 8,7 & 3,7 & 11,3 & 18,4 & 16,0 & 12,1 & 2,2 & 7,8 & 4,4 \\
\hline Mediana & 28,0 & 20,0 & 40,0 & 8,0 & 4,0 & 10,0 & 20,0 & 16,0 & 13,0 & 2,0 & 7,0 & 4,0 \\
\hline Moda & 35,0 & 25,0 & 40,0 & 0,0 & 4,0 & 10,0 & 20,0 & 16,0 & 10,0 & 0,0 & 6,0 & 4,0 \\
\hline Desvio padrão & 7,4 & 11,2 & 20,6 & 6,3 & 2,0 & 7,0 & 6,7 & 0,0 & 5,9 & 2,3 & 5,5 & 1,6 \\
\hline Mínimo & 5,0 & 0,0 & 0,0 & 0,0 & 0,0 & 0,0 & 0,0 & 16,0 & 0,0 & 0,0 & 0,0 & 0,0 \\
\hline Máximo & 35,0 & 40,0 & 60,0 & 20,0 & 8,0 & 26,0 & 26,0 & 16,0 & 22,0 & 9,5 & 28,0 & 8,0 \\
\hline Soma & 2.636 & 1.955 & 3.358 & 860 & 368 & 1.818 & 1.818 & 1.584 & 1.196 & 214,5 & 776 & 436 \\
\hline Máximo possível & 3.465 & 3.960 & 5.940 & 1.980 & 990 & 2.574 & 2.574 & 1.584 & 2.376 & 1.584 & 3.960 & 792 \\
\hline Percentual & 76,1 & 49,4 & 56,5 & 43,4 & 37,2 & 70,6 & 70,6 & 100,0 & 50,3 & 13,5 & 19,6 & 55,1 \\
\hline Coeficiente de variação & 0,28 & 0,57 & 0,61 & 0,73 & 0,54 & 0,37 & 0,37 & 0,00 & 0,49 & 1,07 & 0,70 & 0,40 \\
\hline Percentil 25 & 23,0 & 10,0 & 20,0 & 4,0 & 4,0 & 16,0 & 16,0 & 16,0 & 9,0 & 0,0 & 4,0 & 4,0 \\
\hline Percentil 50 & 28,0 & 20,0 & 40,0 & 8,0 & 4,0 & 20,0 & 20,0 & 16,0 & 13,0 & 2,0 & 7,0 & 4,0 \\
\hline Percentil 75 & 33,0 & 25,0 & 47,0 & 14,0 & 4,0 & 22,0 & 22,0 & 16,0 & 17,0 & 4,0 & 10,0 & 5,0 \\
\hline
\end{tabular}

D/C: Dimensão/Critério; UBS: unidades básicas de saúde.

Nota: Tabela executada no software SPSS (https://www.ibm.com/). Não houve número faltante.

O teste não paramétrico utilizado foi o teste de Wilcoxon para duas amostras independentes. Esse teste se baseia na ordenação do módulo da diferença das duas amostras, o que permite a avaliação $\mu_{A}$ e $\mu_{B}$ :

$$
\left\{\begin{array}{l}
H_{0}: \mu_{A}-\mu_{B}=0 \\
H_{1}: \mu_{A}-\mu_{B} \neq 0
\end{array}\right.
$$

Esse é o teste aplicado para avaliar os indicadores da MAVIL, comparando-se as duas UBS (A e B) pelo total, Dimensão 1, Dimensão 2, Dimensão 3 e critérios da MAVIL.

\section{Teste comparativo de médias}

No resultado do teste para comparar o grau de vínculo das UBS a partir do escore total e das três dimensões, apenas a Dimensão 3, com valor de $\mathrm{p}=0,0051$, apresentou diferença significativa no 
vínculo, rejeitando a hipótese de igualdade entre as duas UBS. O total, Dimensão 1, Dimensão 2, todas com valor de $\mathrm{p} \geq 0,1556$, não rejeitando-se a hipótese de igualdade das UBS a um nível de 5\% de significância.

O resultado do teste para comparar o grau de vínculo das UBS a partir do escore do critério da Dimensão 1 apresentou indicativo de não rejeição da hipótese nula com valor de p = 0,1556, mostrando o mesmo grau de vínculo nessa dimensão nas duas UBS.

Os testes não paramétricos aplicados aos cinco critérios da Dimensão 2 apresentaram valor de $\mathrm{p} \geq$ 0,156, não rejeitando a hipótese nula. O resultado do teste para comparar o grau de vínculo das unidades de saúde a partir dos escores dos critérios da Dimensão 2 não rejeitou a hipótese de igualdade do escore do vínculo entre as UBS para todos os cinco critérios da segunda dimensão, significando que, nessa dimensão, as duas UBS apresentaram o mesmo grau de vínculo.

A análise do resultado do teste para comparar o grau de vínculo das unidades de saúde a partir dos escores dos seis critérios da Dimensão 3 mostra que o Critério 1 (adequação do quantitativo de consultas), o Critério 4 (registro dos exames complementares) e o Critério 6 (registro de aspectos psicossociais) apresentaram diferença significativa entre as UBS, uma vez que obtiveram, no Teste Mann-Whitney, para amostras independentes, significância de 0,000, 0,033 e 0,000, respectivamente. Como os escores calculados para esses critérios da UBS B são maiores que os da UBS A, admitimos melhores resultados para UBS B nesses critérios da terceira dimensão.

\section{Discussão}

O presente artigo avança no processo de validação da MAVIL como instrumento de avaliação dos serviços de APS, no sentido de averiguar sua capacidade de medir o vínculo longitudinal e de captar diferenças entre distintas UBS. Para isso, foram descritas as características do referido atributo em um território de saúde, e foram comparados os resultados das duas UBS desse território. Isso foi possibilitado pelas escolhas estatísticas efetuadas.

A amostra por estrato, junto à opção da soma como métrica, tornou possível a análise descritiva para o território e para cada uma das UBS. Como resultados, observou-se que a pontuação ficou em torno de 51\% para os três recortes do estudo. Quanto às dimensões separadamente, a Dimensão 1 foi a de melhor desempenho, tanto no território quanto para os dois estratos, alcançando mais de $71 \%$ dos pontos possíveis. Essa dimensão apresentou resultados mais coesos, embora tenha tido a ocorrência de entrevistados que não pontuaram (Tabela 1).

A Dimensão 1 ampara-se por dois referenciais: o da territorialização em conjunto à adscrição de clientela, procedente das diretrizes organizativas da APS no SUS 17, e o da utilização de serviços de saúde, resultante da interação entre o indivíduo, que procura os cuidados em vista da percepção da necessidade de saúde, e o profissional, que o conduz dentro do sistema de saúde 28. Portanto, indo ao encontro da APS como ordenadora da atenção, essa dimensão consiste na base sobre a qual a relação interpessoal (Dimensão 2) pode ser estabelecida no âmbito da APS no SUS.

Em comparação com a Dimensão 1, cuja soma dos pontos nos três recortes ultrapassou 70\%, a Dimensão 2, que agrupa elementos que dizem respeito a uma boa relação terapêtica 14, apresentou menores resultados, ficando em torno de $52 \%$ do total de pontos possíveis (Tabelas 1 e 2). Observa-se que, para o critério relativo ao tempo de consulta (Dimensão 2/Critério 4), a performance foi inferior a 48\%. Dos cinco critérios dessa dimensão, apenas o Dimensão 2/Critério 5 (compreensão da fala dos profissionais) alcançou desempenho acima de $60 \%$ nos dois estratos.

Tendo em vista que se trata de um território com 100\% de cobertura populacional pelos serviços de APS e com equipes de saúde da família completas, conforme recomendado pela Política Nacional de Atenção Primária em vigor à época do estudo 17, a expectativa era de melhor performance para a Dimensão 2. Como hipóteses para o resultado, aventam-se duas possibilidades: (i) o desempenho alcançado ser o padrão para a maioria das equipes da ESF/APS; e (ii) o desempenho alcançado estar aquém e requerer intervenções por parte da gestão local com vistas a melhorar aspectos da relação profissional/paciente. Mas, por se tratar de instrumental inédito e ainda sem parâmetros comparativos, outros estudos com ampliação dos territórios amostrados serão necessários para essa qualificação. 
A Dimensão 3, cujo conjunto de seis critérios somam 130 pontos, foi a de pior performance, a despeito de que, nessa dimensão, por conta de o prontuário encontrar-se em plataforma eletrônica, todos os pacientes tenham obtido pontuação máxima no Dimensão 3/Critério 2 (organização adequada do prontuário). Para o desempenho da Dimensão 3, sugerem-se duas hipóteses explicativas: (i) os profissionais da equipe não dão a atenção necessária ao registro em prontuário e (ii) o formato eletrônico, em vez de se constituir em facilidade, por diferentes razões, impõe dificuldades para o registro e a recuperação dos dados 22 .

Vale ressaltar a especificidade da Dimensão 3 para a avaliação da atenção recebida. Embora seja reconhecida a importância dos registros da condição do paciente e da terapêutica adotada, os instrumentos recomendados pela literatura nacional para avaliar a APS, como, por exemplo, as versões do PCATool validadas no Brasil ${ }^{29}$, não contemplam a análise do prontuário. Na MAVIL, a análise dessa dimensão vai ao encontro de autores como Haggerty et al. 30 que veem a continuidade da informação sobre o paciente como condição prévia para a continuidade do cuidado, um dos pilares do vínculo terapêutico. Ademais, embora o desenvolvimento da MAVIL não tenha por principal referência os pilares da qualidade 31, a análise do prontuário aproxima-se do atributo da adequação técnico-científica, posto que averigua a adequação às recomendações do Ministério da Saúde para os pacientes com as condições traçadoras do estudo, que, por sua vez, são pautadas por evidências científicas sob o respaldo de especialistas.

A pesquisa em sua totalidade se propõe a apresentar um instrumental avaliativo dos serviços de APS como opção para aplicação em qualquer território de saúde do país. Por esse motivo, o teste comparativo de médias utilizado precisou cumprir pressupostos teóricos que respaldem aplicações futuras. A aplicação do teste, no caso em questão, rejeitou a hipótese de normalidade. Por esse motivo, optou-se pelo teste não paramétrico de Wilcoxon. Como resultado, para Dimensão 1 e Dimensão 2, não foram apontadas diferenças significativas entre as unidades. No entanto, para Dimensão 3, que se refere à continuidade da informação, dos seis critérios, três apresentaram diferenças significativas.

O fato de a aplicação da MAVIL ter evidenciado diferenças de vínculo, mesmo que em apenas uma das dimensões, indica a capacidade do instrumento em captar diferenças, uma vez que se trata de duas UBS com as mesmas condições de gestão e operação em um mesmo território ${ }^{21}$, cujas populações adscritas possuem características epidemiológicas e sociodemográficas semelhantes. Aperfeiçoar a sensibilidade da MAVIL é processo inerente à continuidade da pesquisa. Nas próximas aplicações da MAVIL que constam como nova etapa da pesquisa, com o aumento da abrangência da amostra pela agregação de outras populações em outros territórios, é possível que surja a indicação da comparação por testes estatísticos paramétricos.

Este artigo teve como foco o estudo das diferenças e semelhanças do vínculo longitudinal identificadas pela MAVIL do ponto de vista quantitativo. A apreciação das diferenças do ponto de vista qualitativo, que consiste em análise dos significados das diferenças encontradas, será objeto de outro artigo.

\section{Considerações finais}

Institucionalizar a avaliação na APS é incorporá-la como atividade regular da gestão dos municípios. Por sua vez, os milhares de municípios do país possuem diferentes realidades sociais, financeiras e de condição de gestão, que podem impor dificuldades nessa incorporação. Disponibilizar um instrumento avaliativo conciso, simples na sua aplicação e na compreensão dos resultados, constitui-se em importante contribuição para a política em discussão. Nesse intuito, a etapa de validação estatística da MAVIL foi realizada.

Todo instrumental avaliativo possui limitações. Nesse caso, há de se considerar que a MAVIL, em seu conjunto de critérios, não contempla ações coletivas e de Vigilância em Saúde no território. Essas ações, que são extremamente relevantes, devem e podem ser monitoradas por indicadores no âmbito da gestão. O propósito da MAVIL é apreciar o espaço e o momento em que acontece o cuidado, bem como analisar as interações e as percepções do paciente, além da adequação técnico-científica do cuidado, a partir da revisão do prontuário. Considera o vínculo, estabelecido com base na confiança no profissional e em respeito à autonomia do sujeito, como atributo que favorecerá a adesão e a continuidade do cuidado. 
Da forma como está estruturada, detalhada em dimensões, critérios e indicadores, a MAVIL possibilita identificar as fragilidades na construção do vínculo terapêutico e, assim, intervir de forma educativa e colaborativa. Ou seja, a MAVIL possibilita identificar fragilidades cuja solução está no escopo de atuação do profissional da atenção, do gerente ou gestor local, sendo assim adequada para avaliar a APS nos municípios.

\section{Colaboradores}

E. M. Cunha contribuiu na concepção do projeto, análise e interpretação dos dados, redação e revisão final. J. M. C. Vargens contribuiu na concepção do projeto, análise e interpretação dos dados e revisão final. M. C. Marques contribuiu na análise e interpretação dos dados e revisão crítica do conteúdo. G. R. B. Andrade contribuiu na revisão crítica relevante do conteúdo intelectual e aprovação da versão final. G. O’Dwyer contribuiu na revisão e aprovação final da versão a ser publicada.

\section{Informações adicionais}

ORCID: Elenice Machado da Cunha (0000-00017471-301X); José Muniz da Costa Vargens (00000002-9297-5213); Marcio Candeias Marques (0000-0002-0566-1209); Gabriela Rieveres Borges de Andrade (0000-0002-1059-5680); Gisele O’Dwyer (0000-0003-0222-1205).

\section{Agradecimentos}

Agradecemos a Brena O'Dwyer Spina da Rosa Machado por ter colaborado como pesquisadora de campo da pesquisa.

\section{Referências}

1. Felisberto E. Da teoria à formulação de uma Política Nacional de Avaliação em Saúde: reabrindo o debate. Ciênc Saúde Colet 2006; 11:553-63.

2. Departamento de Atenção Básica, Secretaria de Atenção à Saúde, Ministério da Saúde. Programa Nacional de Melhoria do Acesso e da Qualidade da Atenção Básica (PMAQ). Brasília: Ministério da Saúde; 2011.

3. Departamento de Atenção Básica, Secretaria de Atenção à Saúde, Ministério da Saúde. Autoavaliação para a Melhoria do Acesso e da Qualidade da Atenção Básica: AMAQ. Brasília: Ministério da Saúde; 2016.

4. Departamento de Saúde da Família, Secretaria de Atenção Primária à Saúde, Ministério da Saúde. Manual do Instrumento de Avaliação da Atenção Primária à Saúde: PCATool-Brasil/2020. Brasília: Ministério da Saúde; 2020.

5. Starfield B. Atenção primária: equilíbrio entre necessidades de saúde, serviços e tecnologia. Brasília: Organização das Nações Unidas para a Educação, a Ciência e a Cultura; Ministério da Saúde; 2002.

6. Leiyu S, Starfield B, Xu J. Validating the Adult Primary Care Assessment Tool. J Fam Pract 2001; 50:161-75.

7. Arce VAR, Sousa MF. Práticas de longitudinalidade no âmbito da Estratégia Saúde da Família no Distrito Federal. Cad Saúde Colet (Rio J.) 2014; 22:62-8.

8. Rubio M, Berg-Weger M, Tebb SS, Lee ES, Rauch S. Objectifying content validity: conducting a content validity study in social work research. Social Work Research 2003; 27:94104. 
9. Martins GA. Sobre confiabilidade e validade. RBGN 2006; 8:1-12.

10. Cunha EM, Andrade GRB, Oliveira CCM, Marques MC, Vargens JMC, O’Dwyer G. Matriz Avaliativa do Vínculo Longitudinal na Atenção Primária: processo de validação por especialistas. Cad Saúde Colet (Rio J.) 2017; 25:249-58.

11. Morosini MVGC, Fonseca AF, Lima LD. Política Nacional de Atenção Básica 2017: retrocessos e riscos para o Sistema Único de Saúde. Saúde Debate 2018; 42:11-24.

12. Ministério da Saúde. Portaria no 2.979, de 12 de novembro de 2019. Institui o Programa Previne Brasil, que estabelece novo modelo de financiamento de custeio da Atenção Primária à Saúde no âmbito do Sistema Único de Saúde, por meio da alteração da Portaria de Consolidação no 6/GM/MS, de 28 de setembro de 2017. Diário Oficial da União 2019; 13 nov.

13. Ministério da Saúde. Portaria no 3.222, de 10 de dezembro de 2019. Dispõe sobre os indicadores do pagamento por desempenho, no âmbito do Programa Previne Brasil. Diário Oficial da União 2019; $11 \mathrm{dez}$.

14. Silva JJC. Vicissitudes do vínculo longitudinal na relação entre médico residente e seu paciente na Atenção Primária à Saúde [Dissertação de Mestrado]. Rio de Janeiro: Universidade Federal do Rio de Janeiro; 2019.

15. Cunha EM. Vínculo longitudinal na atenção primária: avaliando os modelos assistenciais do SUS [Tese de Doutorado]. Rio de Janeiro: Escola Nacional de Saúde Pública Sergio Arouca, Fundação Oswaldo Cruz; 2009.

16. Quivy R, Van Campenhoudt L. Manual de investigação em ciências sociais. 2a Ed. Lisboa: Editora Gradiva; 1998.

17. Ministério da Saúde. Portaria no 2.488, de 21 de outubro de 2011. Aprova a Política Nacional de Atenção Básica, estabelecendo a revisão de diretrizes e normas para a organização da Atenção Básica, para a Estratégia Saúde da Família (ESF) e o Programa de Agentes Comunitários de Saúde (PACS). Diário Oficial da União 2011; 24 out.

18. Cunha EM, Giovanella L. Longitudinalidade/ continuidade do cuidado: identificando dimensões e variáveis para a avaliação da Atenção Primária no contexto do sistema público de saúde brasileiro. Ciênc Saúde Colet 2011; 16 Suppl 1:1029-42.

19. Kessner DM, Kalk CE, Singer J. Evaluacion de la calidad de la salud por el metodo de los procesos trazadores. In: Organização Panamericana de la Salud, editor. Investigaciones sobre servicios de salud: una antologia. Washington DC: Organização Panamericana de la Salud; 1992. p. 555-63. (Publicación Científica 534).
20. Informe Ensp. Teias atinge 100\% de cobertura da saúde da família em Manguinhos. http:// www.ensp.fiocruz.br/portal-ensp/informe/ site/materia/detalhe/23285 (acessado em 27/ Mai/2020).

21. Fundação para o Desenvolvimento Científico e Tecnológico em Saúde. Contrato de Gestão no 32/2014- celebrado entre a FIOTEC e a Secretaria Municipal de Saúde do Rio de Janeiro. http://www.fiotec.fiocruz.br/projetos/contra to-de-gestao (acessado em 16/Mar/2020).

22. Gonçalves JMS. As promessas das TICs para a gestão do SUS: uma reflexão sociotécnica sobre a implantação de um software para a Estratégia de Saúde da Família [Dissertação de Mestrado]. Rio de Janeiro: Instituto Alberto Luiz Coimbra de Pós-Graduação e Pesquisa de Engenharia, Universidade Federal do Rio de Janeiro; 2016.

23. Souza-Junior PRB, Freitas MPS, Antonaci GA, Szwarcwald CL. Desenho da amostra da Pesquisa Nacional de Saúde 2013. Epidemiol Serv Saúde 2015; 24:207-16.

24. Instituto Brasileiro de Geografia e Estatística. Metodologia do Censo Demográfico 2000. Rio de Janeiro: Instituto Brasileiro de Geografia e Estatística; 2003.

25. Vasconcellos MTL. Comentário: Eunice Pinho de Castro Silva, o despontar da pesquisa por amostragem na saúde pública brasileira. Rev Saúde Pública 2006; 40:769-71.

26. Cochran W. Sampling techiniques. 3rd Ed. New York: Editora John Wiley \& Sons; 1977.

27. Bolfarine H, Bussa BWO. Elementos de amostragem. São Paulo: Editora Edgrard Blucher; 2005.

28. Travassos C, Martins M. Uma revisão sobre os conceitos de acesso e utilização de serviços de saúde. Cad Saúde Pública 2004; 20 Suppl 2:S190-8.

29. Harzheim E, Gonçalves MR, D’Avila OP, Hauser L, Pinto LF. Estudos de PCATool no Brasil. In: Mendonça MHM, Matta GC, Gondim R, Giovanella L, organizadores. Atenção primária à saúde no Brasil: conceitos, práticas e pesquisa. Rio de Janeiro: Editora Fiocruz; 2018. p. 493-525.

30. Haggerty JL, Reid RJ, Freeman GK, Starfield BH, Adair CE, McKendry R. Continuity of care: a multidisciplinary review. BMJ 2003; 327:1219-21.

31. Frias PG, Costa JMBS, Figueiró AC, Mendes MFM, Vidal SA. Atributos da qualidade em saúde. In: Samico I, Felisberto E, Figueiró AC, Frias PG, organizadores. Avaliação em saúde: bases conceituais e operacionais. Rio de Janeiro: MedBook; 2010. p. 43-55. 


\section{Abstract}

In keeping with the process of institutionalization of evaluation of primary healthcare in Brazil, we developed the Evaluative Matrix of the Longitudinal Bond (MAVIL in Portuguese), a simple and concise evaluative tool. MAVIL is structured according to three dimensions in the concept of longitudinal bond: Dimension 1 - recognition of the basic health unit (UBS in Portuguese) as a regular source of care, Dimension 2 - healthcare worker/ patient interpersonal relationship, and Dimension 3 - continuity of information. The instrument consists of a structured set of 12 criteria, 20 indicators and respective questions, and verification items submitted to various validation stages. The current article presents the statistical validation of the MAVIL application in two UBS in a health territory in order to support future applications and also verify the instrument's capacity to capture differences in the longitudinal bond profile between UBS. The method included the following definitions: sample design, choice of the metric, descriptive data analysis, and comparative test of means, through application of the test of normality and nonparametric statistic. As results, the sample by strata, together with option of totaling the points from the MAVIL items as the metric, allowed describing the longitudinal bond profile for three cross-sections: territory and UBS A and B. To verify the capacity of MAVIL to capture differences in the longitudinal bond profile, we tested the hypothesis of the data's normality with the Kolmogorov-Smirnov test. Having rejected the hypothesis of normality, we opted for the Wilcoxon nonparametric test to compare the UBS. Dimension 1 and Dimension 2 showed similar profiles, but there was a significant difference for Dimension 3, attesting to the sensitivity of MAVIL.

Primary Health Care; Validation Study; Health Services Research; Health Manegement

\section{Resumen}

En consonancia con el proceso de institucionalización de la evaluación de la atención primaria en Brasil, se desarrolló la Matriz Evaluativa del Vinculo Longitudinal (MAVIL), un instrumento evaluativo simple y conciso. MAVIL se estructura a partir de las tres dimensiones del concepto de vínculo longitudinal: Dimensión 1 - reconocimiento de la unidad básica de salud (UBS) como fuente regular de cuidados, Dimensión 2 - relación interpersonal profesional/paciente y Dimensión 3 - continuidad de la información. Consiste en un conjunto estructurado de 12 criterios, 20 indicadores y sus respectivas cuestiones e items de verificación, sometido a varias etapas de validación. Este artículo presenta la validación estadística de la aplicación MAVIL en dos UBS de una zona con servicios de salud, con el fin de respaldar sus futuras aplicaciones y también averiguar la capacidad de la herramienta para captar diferencias en el perfil del vínculo longitudinal entre UBS. El método consistió en las siguientes definiciones: diseño de la muestra, elección del sistema de medición, análisis descriptivo de los datos y test comparativo de medias, a través de la aplicación del test de normalidad y de estadística no paramétrica. Como resultados: muestra por estrato, junto a la opción de suma de los puntos de los items de la MAVIL como sistema de medición, posibilitó describir el perfil del vínculo longitudinal para las tres secciones elegidas: territorio, UBS A y B. Para verificar la capacidad de la MAVIL en captar la diferencia en el perfil de vínculo longitudinal se probó la hipótesis de la normalidad de los datos, a partir del test de Kolmogorov-Smirnov. Rechazada la hipótesis de la normalidad, se optó por la prueba no paramétrica de Wilcoxon para comparar las UBS. En Dimensión 1 y Dimensión 2 se constató la semejanza de perfil, sin embargo, en la Dimensión 3 hubo una diferencia significativa, constatando la sensibilidad de la MAVIL.

Atención Primaria de Salud; Estudio de Validación; Investigación sobre Servicios de Salud; Gestion en Salud
Recebido em 09/Jul/2020

Versão final reapresentada em 11/Nov/2020

Aprovado em 02/Dez/2020 